\title{
CLOSED CONVEX HYPERSURFACES WITH CURVATURE RESTRICTIONS
}

\author{
ROLF SCHNEIDER
}

(Communicated by David G. Ebin)

\begin{abstract}
Using elementary facts from the theory of convex bodies, we obtain an improved version of J. Rauch's [5] inclusion theorem for ovaloids with comparable second fundamental forms and a generalization of a result of A. W. Goodman [4] on the position of the curvature ceritroids for ovaloids with restricted radii of curvature.
\end{abstract}

1. Introduction. For a convex curve $C$ in the plane and a point $z$ in its interior, we denote by $D_{1}(z)$ the smallest and by $D_{2}(z)$ the greatest distance of the points of $C$ from $z$. Let $C$ be of class $C^{2}$ and suppose that its radius of curvature function $\rho$ satisfies $0<R_{1} \leq \rho \leq R_{2}$ with some constants $R_{1}, R_{2}$. If $z$ is the perimeter centroid of $C$, then Goodman [4] proved that $R_{1} \leq D_{1}(z) \leq D_{2}(z) \leq R_{2}$. He asked if here $z$ may be replaced by Steiner's curvature centroid. In the present note we obtain a general result of this kind, valid for ovaloids in $\mathbb{R}^{d}$ and for the whole series of area and curvature centroids (Theorem 2). In its proof we use an improved version of a theorem of Rauch [5] (Theorem 1 below). Our main aim is to make better known the fact that assertions of this kind can be given a stronger form (and a simpler proof) if some elementary results from the theory of convex bodies are used.

2. Ovaloids with comparable second fundamental forms. By an ovaloid we understand a closed convex hypersurface $M$ in $\mathbb{R}^{d}(d \geq 2)$ of class $C^{2}$ and with everywhere positive curvatures. For $x \in M$, we denote by $n(x)$ the outward unit normal vector to $M$ at $x$. The tangent space $M_{x}$ of $M$ at $x$ is identified with the linear subspace of $\mathbb{R}^{d}$ orthogonal to $n(x)$. The Weingarten map $L_{x}: M_{x} \rightarrow M_{x}$ is defined by $L_{x} v=D_{v} n(x)$ for $v \in M_{x}$, where $D_{v}$ denotes the directional derivative operator (as in [5]). The second fundamental form of $M$ at $x$ is given by $\operatorname{II}_{x}(v)=$ $\left\langle L_{x} v, v\right\rangle$ for $v \in M_{x}$. For $u \in \mathbb{R}^{d} \backslash\{0\}$ we denote by $p(u)$ the unique point of $M$ with $n(p(u))=u /\|u\|$. Since the Gauss map of $M$ and its inverse are of class $C^{1}$, the function $p$ thus defined is of class $C^{\mathbf{1}}$. If we restrict this function to the unit sphere $\Omega=\left\{u \in \mathbb{R}^{d}:\|u\|=1\right\}$, we have $D_{v} p(u)=\left(L_{p(u)}\right)^{-1} v[\mathbf{5}$, p. 502].

The ovaloid $M$ is the boundary of a convex body $K$. Its support function is defined by

$$
H(u)=\max _{y \in K}\langle y, u\rangle \quad \text { for } u \in \mathbb{R}^{d},
$$

which implies $H(u)=\langle p(u), u\rangle$ and hence

$$
\operatorname{grad} H(u)=p(u)
$$

Received by the editors July 22, 1987.

1980 Mathematics Subject Classification (1985 Revision). Primary 53A07; Secondary 52A20. 
(cf. [2, p. 26]). It follows that $H$ is of class $C^{2}$. Let $d^{2} H_{u}$ be the second differential of $H$ at $u$, considered as a bilinear form on $\mathbb{R}^{d}$. For $u \in \mathbb{R}^{d} \backslash\{0\}$ we denote by $\pi_{u}$ the orthogonal projection onto the orthogonal complement of $u$.

LEMMA. $d^{2} H_{u}(a, b)=\|u\|^{-1}\left\langle\left(L_{p(u)}\right)^{-1} \pi_{u} a, \pi_{u} b\right\rangle$ for $a, b \in \mathbb{R}^{d}$.

PROOF. By homogeneity, we may assume $\|u\|=1$. Let $\left(e_{1}, \ldots, e_{d}\right)$ be an orthonormal basis for $\mathbb{R}^{d}$ with $e_{d}=u$. We write $\left(y^{1}, \ldots, y^{d}\right)$ for the coordinates of $y \in \mathbb{R}^{d}$ and $H_{i j}$ for the second partial derivatives of $H$ with respect to this basis. The homogeneity relation

$$
\sum_{j=1}^{d} H_{i j}(y) y^{j}=0 \quad \text { for } i=1, \ldots, d
$$

yields $H_{i d}(u)=0$ and hence

$$
d^{2} H_{u}(a, b)=\sum_{i, j=1}^{d-1} H_{i j}(u) a^{i} b^{j} \quad \text { for } a, b \in \mathbb{R}^{d}
$$

On the other hand, $\left\langle\left(L_{p(u)}\right)^{-1} e_{i}, e_{j}\right\rangle=\left\langle D_{e_{i}} p(u), e_{j}\right\rangle$ is the $j$ th coordinate of $D_{e_{i}} p(u)$ and hence by $(2.1)$ equal to $H_{j i}(u)$, if $i, j \leq d-1$. This gives

$$
\left\langle\left(L_{p(u)}\right)^{-1} \pi_{u} a, \pi_{u} b\right\rangle=\sum_{i, j=1}^{d-1} H_{i j}(u) a^{i} b^{j},
$$

which proves the lemma.

Now let $\tilde{M}$ be a second ovaloid. All objects defined for $\tilde{M}$ as above for $M$ are distinguished by a tilde.

THEOREM 1. If $M$ and $\tilde{M}$ are ovaloids such that $\mathrm{II}_{x} \geq \widetilde{\mathrm{II}}_{\tilde{x}}$ for all points $x \in M$, $\tilde{x} \in \tilde{M}$ where the outer normal vectors to $M$ and $\tilde{M}$ are the same, then there exists a convex body $L$ with $\tilde{K}=K+L$.

PrOOF. The assumption $\mathrm{II}_{x} \geq \widetilde{\mathrm{II}}_{\tilde{x}}$, or $L_{x} \geq \tilde{L}_{\tilde{x}}$, for $n(x)=\tilde{n}(\tilde{x})$ implies $\left(L_{p(u)}\right)^{-1} \leq\left(\tilde{L}_{\tilde{p}(u)}\right)^{-1}\left[\mathbf{5}\right.$, p. 503], hence the lemma yields $d^{2} H_{u} \leq d^{2} \tilde{H}_{u}$ for all $u \neq 0$. Thus the function $\tilde{H}-H$ has positive semidefinite Hessian on $\mathbb{P}^{d} \backslash\{0\}$ and is, therefore, convex. Being positively homogeneous of degree 1 , it is the support function $H_{L}$ of a uniquely determined convex body $L[2$, p. 26], and the equation $\tilde{H}=H+H_{L}$ is equivalent to $\tilde{K}=K+L[2$, p. 29]. The proof is complete.

Under the assumptions of Theorem 1, Rauch [5] has shown that $M$ can fit inside $\tilde{M}$. This is an immediate consequence of our strengthened version: Each point $t \in L$ satisfies $M+t \in \tilde{K}$. The theorem as formulated by Rauch also follows: Suppose that $M, \tilde{M}$ satisfy the assumptions above and are internally tangent at some point $x$ (i.e., $x \in M \cap \tilde{M}$ and $n(x)=\tilde{n}(x)=u$, say). The point $x \in \tilde{K}=K+L$ has a representation $x=x_{1}+x_{2}$ with $x_{1} \in K$ and $x_{2} \in L$. From $\tilde{H}(u)=\langle x, u\rangle=$ $\left\langle x_{1}, u\right\rangle+\left\langle x_{2}, u\right\rangle \leq H(u)+H_{L}(u)=\tilde{H}(u)$ we conclude that $x_{1}=x$ and hence $0 \in L$, which implies $M \subset K \subset \tilde{K}$. 
3. The position of the curvature centroids. We generalize the result of Goodman [4]. The $r$ th curvature centroid of the ovaloid $M$ is defined by

$$
p_{r}(M)=\frac{\int_{M} x H_{r-1}(x) d A(x)}{\int_{M} H_{r-1} d A}, \quad r=1, \ldots, d,
$$

where $H_{k}$ denotes the $k$ th elementary symmetric function of the principal curvatures of $M$ and $d A$ is the surface area element. In particular, $p_{1}$ is the area centroid and $p_{d}$ is Steiner's curvature centroid (for more information on curvature centroids, see [6]). By $B(z, R)$ we denote the closed ball with centre $z$ and radius $R>0$.

THEOREM 2. Let $M$ be an ovaloid in $\mathbb{R}^{d}$ and let $R_{1}, R_{2}$ be constants such that $0<R_{1} \leq \rho \leq R_{2}$ whenever $\rho$ is a radius of curvature of $M$. Let $K$ be the convex body bounded by $M$. If $z$ is any of the curvature centroids $p_{1}(M), \ldots, p_{d}(M)$, then $B\left(z, R_{1}\right) \subset K \subset B\left(z, R_{2}\right)$.

PROOF. For $R>0$ let $\operatorname{II}(R)$ denote the second fundamental form of the sphere $R \Omega$. The assumptions of Theorem 2 imply that the second fundamental form II of $M$ satisfies $\mathrm{II}_{u}\left(R_{1}\right) \geq \mathrm{II}_{p(u)} \geq \mathrm{II}_{u}\left(R_{2}\right)$. From Theorem 1 (which in this special case reduces to a known argument, cf. Firey [3] or Weil [7]) we deduce the existence of convex bodies $K_{1}, K_{2}$ satisfying

$$
\begin{aligned}
& K=R_{1} B+K_{1}, \\
& R_{2} B=K+K_{2},
\end{aligned}
$$

where $B$ denotes the unit ball of $\mathbb{R}^{d}$. For a strictly convex body $L$ and for $u \in \Omega$, we now write $p(L, u)$ for the unique point of the boundary $\partial L$ where $u$ is attained as outward unit normal vector. From (2.1), (3.1), (3.2) we have

$$
\begin{aligned}
& p(K, u)=R_{1} u+p\left(K_{1}, u\right), \\
& R_{2} u=p(K, u)+p\left(K_{2}, u\right) .
\end{aligned}
$$

Given any positive Borel measure $\varphi$ on $\Omega$ which satisfies

$$
\int_{\Omega} d \varphi=1 \text { and } \int_{\Omega} u d \varphi(u)=0
$$

we may define the $\varphi$-centroid of $L$ by

$$
c(L)=\int_{\Omega} p(L, u) d \varphi(u) .
$$

Since $\varphi$ is nonnegative and normalized and since $L$ is convex, we have $c(L) \in L$. Now (3.3) yields $c(K)=c\left(K_{1}\right) \in K_{1}$, hence (3.1) implies $K \supset R_{1} B+c(K)$, thus $B\left(c(K), R_{1}\right) \subset K$. Similarly, (3.4) yields $0=c(K)+c\left(K_{2}\right)$, hence $-c(K) \in K_{2}$, and (3.2) implies $R_{2} B \supset K-c(K)$, thus $K \subset B\left(c(K), R_{2}\right)$. It remains to observe that the measure $\varphi$ defined by

$$
d \varphi=\frac{S_{n-r} d \omega}{\int_{M} H_{r-1} d A},
$$

where $S_{k}(u)$ is the $k$ th elementary symmetric function of the principal radii of curvature of $M$ at $p(K, u)$ and $\omega$ is spherical Lebesgue measure, satisfies (3.5) and gives $c(K)=p_{r}(M)$ (cf. [6, pp. 122-123]). This completes the proof of Theorem 2 . 
Finally we remark that the left-hand inclusion of Theorem 2 remains true if $z$ is the centroid $p_{0}(K)$ (centre of mass of $K$ ). This follows from the fact that $p_{0}(K)=$ $p_{0}\left(R_{1} B+K_{1}\right)$ is a convex combination of the points $p_{0}\left(K_{1}\right), p_{1}\left(\partial K_{1}\right), \ldots, p_{d}\left(\partial K_{1}\right)$ (see [6, formula (27)]) and hence a point of $K_{1}$ (this remark extends an argument of Blaschke [1]).

\section{REFERENCES}

1. W. Blaschke, Über die Schwerpunkte von Eibereichen, Math. Z. 36 (1933), 166.

2. T. Bonnesen and W. Fenchel, Theorie der konvexen Körper, Springer-Verlag, Berlin, 1934.

3. W. J. Firey, Inner contact measures, Mathematika 26 (1979), 106-112.

4. A. W. Goodman, Convex curves of bounded type, Internat. J. Math. Math. Sci. 8 (1985), 625-633.

5. J. Rauch, An inclusion theorem for ovaloids with comparable second fundamental forms, J. Differential Geom. 9 (1974), 501-505.

6. R. Schneider, Krümmungsschwerpunkte konvexer Körper. I, Abh. Math. Sem. Univ. Hamburg 37 (1972), 112-132.

7. W. Weil, Inner contact probabilities for convex bodies, Adv. Appl. Prob. 14 (1982), 582-599.

Mathematisches Institut, Albert-Ludwigs-Universität, D-7800 Freiburg, FEDERAL REPUBLIC OF GERMANY 